\title{
Acknowledgment to Reviewers of ChemEngineering in 2020
}

\author{
ChemEngineering Editorial Office
}

MDPI AG, St. Alban-Anlage 66, 4052 Basel, Switzerland

Citation: ChemEngineering Editorial

Office. Acknowledgment to

Reviewers of ChemEngineering in

2020. ChemEngineering 2021, 5, 4.

https://doi.org/10.3390/

chemengineering 5010004

Published: 19 January 2021

Publisher's Note: MDPI stays neutral with regard to jurisdictional claims in published maps and institutional affiliations.

Copyright: (C) 2021 by the author. Licensee MDPI, Basel, Switzerland. This article is an open access article distributed under the terms and conditions of the Creative Commons Attribution (CC BY) license (http://creativecommons.org/licenses/by/4.0/).

Arbabi, Vahid
Atta-Obeng, Emmanuel
Bahrami, Amin
Bakhshian, Sahar
Barroso-Solares, Suset
Beisl, Stefan
Benedek, Csilla
Bhagia, Samarthya
Blais, Bruno
Böker, Kai O.
Bück, Andreas
Buijs, Wim
Camposeo, Andrea
Carreon, Moises
Cecone, Claudio
Chiang, Ming-His
Cota, Iuliana
Crowley, Shane V.
Czekała, Wojciech
Dama, Murali
Dangelo, Anthony
Das, Gautam
Diederichsen, Kyle
Dimitrellou, Dimitra
Distler, Petr
Dorado, Antonio
Drzeżdż, Joanna
Egedy, Attila
Favergeon, Loïc
Fresner, Johannes
Fu, Guopeng
Fu, Yucheng
Fujita, Toyohisa
Galkin, Maxim
Garcia-Salaberri, Pablo A.

\author{
Gargiulo, Valentina \\ Geier, Martin \\ González, Begoña \\ Gostomski, Peter \\ Gunawan, Andrey \\ Güttel, Robert \\ Hart, Kyle \\ Hebishy, Essam \\ Hełdak, Maria \\ Hintermair, Ulrich \\ Ho, Kacie \\ Holmberg, Henrik \\ Hong, Min \\ Ierapetritou, Marianthi \\ Ionut, Ioana A. \\ Isalgue, Antonio \\ Islam, Nahidul \\ Janneck, Eberhard \\ Javadi, Ardalan \\ Jeon, Ju-Won \\ Josephson, Alexander J. \\ Kalmár, József \\ Kaluža, Luděk \\ Khosravani, Mohammad Reza \\ Kontoravdi, Cleo \\ Kozlovskiy, Artem \\ Kristóf, Tamás \\ Kucharska, Karolina \\ Kuttner, Christian \\ Kvasha, Andriy \\ Lee, Changhee \\ Lee, Woo Hyoung \\ Lewandowska, Katarzyna \\ Liang, Peishih \\ Liu, Yuan
}


Liu, Zongyuan

López De Leon, Luis R.

Maciej, Marek

Maknickas, Algirdas

Marakatti, Vijaykumar

Martín, Beatriz De Rivas

Martinelli, Michela

Martins Morgado, Teresa Leonor

Marzoughi, Omid

Melinte, Violeta

Meng, Jianping

Michalska, Anna

Mierzwa, Dominik

Milanesio, Marco

Mukherjee, Rajib

Nanjundaswamy, Ananda

Nemchinova, Nina

Nhuchhen, Daya

Nobile, Lucio

Nogueira, Carlos Alberto Gonçalves

Oancea, Florin

Odeh, Saad

Ostanek, Jason

Papagni, Antonio

Penkova, Nina

Pereira, Carlos Dias

Petković, Gorana

Porcarelli, Luca

Putta, Koteswara Rao

Qiao, Pengfei

Reynier, Nicolas

Richtera, Lukáš

Rizzolo, Anna

Rodríguez, Camino Fernández

Rodríguez, Olga

Romdhana, Hedi

Rother, Gernot

Saidani-Scott, Hind

Sancaktar, Erol

Sarniak, Mariusz

Sęk, Jerzy

Senadeera, Wijitha
Sengupta, Saptarshi

Shafer, Wilson D.

Shimosako, Naoki

Shorstkii, Ivan

Soco, Eleonora

Soleimani, Majid

Soler, Jaime

Spiridigliozzi, Luca

Stawiński, Wojciech

Stelescu, Maria Daniela

Sukin, Ivan

Szaleniec, Maciej

Szekalska, Marta

Szurgacz, Dawid

Szymczyk, Magdalena

Tade, Moses

Tafuni, Angelo

Taherdangkoo, Reza

Tang, Yu-Hang

Teo, Nicholas

Tomoyoshi, Nishimura

Trinh, Thuat

Trofimova, Maya

Tronci, Stefania

Tsvetkov, Nikolai

Valero-Lara, Pedro

Vanyorek, László

Viganò, Federico

Vilgis, Thomas

Villacorta, Byron

Wehinger, Gregor

Wiącek, Joanna

Witz, Christian

Wycisk, Ryszard

Xiao, Xinxin

Xie, Weiguo

Yoshida, Kazunari

Zhang, Xin

Zhang, Xueqiang Alex

Zhang, Yanlin

Zhu, Taishan Zhu 